9. W. Rudin, Measure algebras on abelian groups, Bull. Amer. Math. Soc. 65 (1959), 227-247.

10. G. E. Silov, On decomposition of a commutative normed ring in a direct sum of ideals, Mat. Sb. 32 (1953), 353-364.

11. A. Weil, L'intégration dans les groupes topologiques et ses applications, Actualités Sci. Ind. no. 869, Hermann, Paris, 1940.

12. J. Wermer, Ideals in a class of commutative Banach algebras, Duke Math. J. 20 (1953), 273-278.

Ohio State University and

UNiversity of Notre DaMe

\title{
A MULTI-POINT GENERALIZATION OF NEWTON'S DIVIDED DIFFERENCE FORMULA
}

\section{HERBERT E. SALZER}

Newton's divided difference formula for the polynomial of degree $n-1$ determined by the values of the function $f(x)$ and its divided differences up to the $(n-1)$ th order originating from any single point, has the following multi-point generalization:

Theorem I. The unique interpolation polynomial $P(x)$, of degree $\sum_{i=1}^{n} r_{i}-1$, which is determined by $f\left(x_{i}\right) \equiv f_{i} \equiv D^{0} f_{i}$ and the first $r_{i}-1$ divided differences, $\mathfrak{D}^{m} f_{i} \equiv\left[x_{i} x_{i_{1}} \cdots x_{i_{m}}\right], m=1, \cdots, r_{i}-1$, originating from the $n$ points $x_{i}, i=1, \cdots, n$, is expressible as

(1) $\quad P(x)=\sum_{i=1}^{n} \Pi_{i}(x) \psi_{i}(x)$, where

(2) $\quad \Pi_{i}(x)=\prod_{j=1, j \neq i}^{n}\left(x-x_{j}\right)\left(x-x_{j_{1}}\right) \cdots\left(x-x_{j_{r_{j}-1}}\right)$,

(3) $\quad \psi_{i}(x)=\sum_{m=0}^{r_{i}-1}\left[x_{i} x_{i_{1}} \cdots x_{i_{m}}\right] \psi_{i, m}(x)$, and

(4) $\psi_{i, m}(x)=\sum_{s=0}^{r_{i}-m-1}\left[x_{i_{m}} x_{i_{m+1}} \cdots x_{i_{m+}}\right]_{1 / \mathbb{I}_{i}}\left(x-x_{i}\right)\left(x-x_{i_{1}}\right) \cdots$

$$
\left(x-x_{i_{m+1-1}}\right) .
$$

In (3) and (4), $x_{i_{0}} \equiv x_{i} ;$ in (3), $\left[x_{i}\right] \equiv f_{i} ;$ in (4), $\left[x_{i_{m}}\right]_{1 / \Pi_{i}} \equiv 1 / \Pi_{i}\left(x_{i_{m}}\right)$, the sth order divided difference $\left[x_{i_{m}} x_{i_{m+1}} \cdots x_{i_{m+l}}\right]_{1 / \mathrm{I}_{i}}$ is for the function $1 / \Pi_{i}(x)$, and $\psi_{i, 0}(x)$ begins $\left[x_{i}\right]_{1 / \Pi_{i}}+\cdots$.

Presented to the Society, April 22, 1961; received by the editors February 23, 1961. 
Theorem I is a generalization of the author's previous result involving advancing differences of $f(x)$, at equal intervals, at each $x_{i}$ [1]. Also, Theorem I extends the result in [1] beyond generalization to irregular distribution of points $\left(x_{i}, x_{i_{1}}, \cdots, x_{i_{r_{i}-1}}\right)$ in that the coefficients of $\left(x-x_{i}\right)\left(x-x_{i_{1}}\right) \cdots\left(x-x_{i_{m+\ell-1}}\right)$ in (4) are given explicitly as $\left[x_{i_{m}} x_{i_{m+1}} \cdots x_{i_{m+}}\right]_{1 / \Pi_{i}}$, whereas in [1] corresponding quantities that are proportional to those coefficients are given implicitly as solutions of a triangular linear system.

Theorem I, which may be regarded as a partitioned form of Newton's divided difference formula, might be applied advantageously, say in reducing the computing error by employing divided differences of lower orders, or where the points $\left(x_{i}, x_{i_{1}}, \cdots, x_{i_{r_{i}-1}}\right)$ are at the same irregular intervals, so that the divided differences $\left[x_{i_{m}} x_{i_{m+1}} \cdots x_{i_{m+\ell}}\right]_{1 / \Pi_{i}}$ are the same linear combinations of $1 / \Pi_{i}\left(x_{i_{m+\ell}}\right)$, $t=0,1, \cdots, s$, for every $i$.

Proof of Theorem I. We use the following formula [2] for the $m$ th divided difference of any $F(x)$ for the $m+1$ points $x_{0}, x_{1}, \cdots, x_{m}$ :

$$
\mathfrak{D}^{m} F \equiv\left[x_{0} x_{1} \cdots x_{m}\right]_{F}=\sum_{i=0}^{m}\left\{F\left(x_{i}\right) / \prod_{j=0, j \neq i}^{m}\left(x_{i}-x_{j}\right)\right\} .
$$

To show $\left.\mathfrak{D}^{m} P(x)\right|_{x-x_{i}}=\left[x_{i} x_{i_{1}} \cdots x_{i_{m}}\right], m=0,1, \cdots, r_{i}-1$, we note first from (5) and (2) that the divided difference operator $\left.D^{m} \cdots\right|_{x=x_{i}}$ annihilates every term in (1) except the $i$ th. It suffices to show that

$$
\left.D^{m^{\prime}} \Pi_{i}(x) \psi_{i, m}(x)\right|_{x=x_{i}}=\delta_{m}^{m^{\prime}}, \quad m^{\prime}=0,1, \cdots, r_{i}-1 .
$$

For $m^{\prime} \leqq m$, the result follows from (4) and (5), for arbitrary values of the coefficients of $\left(x-x_{i}\right)\left(x-x_{i_{1}}\right) \cdots\left(x-x_{i_{m+\ell-1}}\right)$ in (4) beyond the first.

We prove (6) for any $m^{\prime}>m$ by induction, assuming (4) as far as $\left[x_{i_{m}} x_{i_{m+1}} \cdots x_{i_{m+\ell-1}}\right]_{1 / \Pi_{i}}\left(x-x_{i}\right)\left(x-x_{i_{1}}\right) \cdots\left(x-x_{i_{m+\ell-2}}\right)$ to be equivalent to (6) for $m^{\prime} \leqq m+s-1$, which was just shown for $s=1$. For (6) to hold when $m^{\prime}=m+s$, denoting in (4) the coefficient of $\left(x-x_{i}\right)\left(x-x_{i_{1}}\right) \cdots\left(x-x_{i_{m+s-1}}\right)$ by $a_{i_{m}}^{(s)}$, application of (5) to $\left[x_{i} x_{i_{1}} \cdots x_{i_{m}} \cdots x_{i_{m+}}\right]_{\Pi_{i} \psi_{i, m}}$ should give

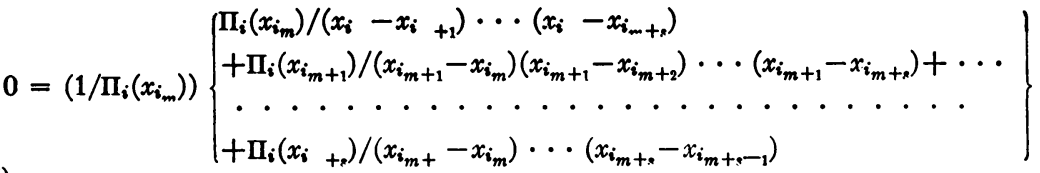

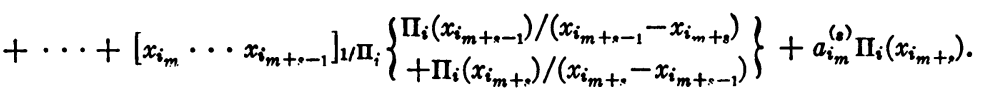


The quantities in braces in (7) are

$$
\left[x_{i_{m}} \cdots x_{i_{m+\varepsilon}}\right]_{\Pi_{i}}, \cdots,\left[x_{i_{m+\ell-1}} x_{i_{m+\varepsilon}}\right]_{\Pi_{i}} \text {. }
$$

We now employ the Popoviciu-Steffensen theorem for the $m$ th divided difference of the product $f(x) \phi(x)$ in terms of the divided differences of $f(x)$ and $\phi(x)[3 ; 4]:^{1}$

$$
\left[x_{0} x_{1} \cdots x_{m}\right]_{f \phi}=\sum_{i=0}^{m}\left[x_{0} \cdots x_{i}\right]_{f}\left[x_{i} \cdots x_{m}\right]_{\phi} .
$$

Applying (8) to the product $1 \equiv\left\{1 / \Pi_{i}(x)\right\} \Pi_{i}(x)$ for the points $\left(x_{i_{m}}, x_{i_{m+1}}, \cdots, x_{i_{m+}}\right)$, we have

$$
\begin{aligned}
0= & \left(1 / \Pi_{i}\left(x_{i_{m}}\right)\right)\left[x_{i_{m}} \cdots x_{i_{m+\ell}}\right]_{\Pi_{i}}+\cdots \\
& +\left[x_{i_{m}} \cdots x_{i_{m+\ell-1}}\right]_{1 / \Pi_{i}}\left[x_{i_{m+\ell-1}} x_{i_{m+}}\right]_{\Pi_{i}} \\
& +\left[x_{i_{m}} \cdots x_{i_{m+\ell}}\right]_{1 / \Pi_{i}} \Pi_{i}\left(x_{i_{m+\ell}}\right)
\end{aligned}
$$

Comparison of (9) with (7) shows $a_{i_{m}}^{(s)}=\left[x_{i_{m}} \cdots x_{i_{m+\varepsilon}}\right]_{1 / \Pi_{i}}$.

The uniqueness of $P(x)$ is apparent, since specification by $f_{i}$, $D f_{i}, \cdots, D^{r_{i}-1} f_{i}, i=1, \cdots, n$, is equivalent to specification by certain $\sum_{i=1}^{n} r_{i}$ functional values $f_{i}, f_{i_{1}}, \cdots, f_{i_{r_{i}-1}}$ at $x_{i}, x_{i_{1}}, \cdots, x_{i_{r_{i}-1}}$, for which the Lagrange interpolation polynomial of degree $\sum_{i=1}^{n} r_{i}-1$ (a rearrangement of $P(x)$ ) is unique.

\section{REFERENCES}

1. H. E. Salzer, Hermite's general osculatory interpolation formula and a finite difference analogue, J. Soc. Indust. Appl. Math. 8 (1960), 18-27.

2. L. M. Milne-Thomson, The calculus of finite differences, Macmillan, London, 1933, p. 7.

3. T. Popoviciu, Sur quelques proprietés des fonctions d'une ou de deux variables reelles, Mathematica 8 (1934), 1-85.

4. J. F. Steffensen, Note on divided differences, Danske Vid. Selsk. Math.-Fys. Medd. 17, no. 3 (1939), 12 pp.

General Dynamics/Astronautics

${ }^{1}$ Popoviciu published a statement of (8) without proof in his thesis [3], apparently unknown to Steffensen whose proof appeared 5 or 6 years later [4]. 